\title{
A Comparison of Reliability, Delay and \\ Complexity for Standalone Cognitive Radio Spectrum Sensing Schemes
}

Xing Liu, Barry G. Evans and Klaus Moessner, University of Surrey

\begin{abstract}
The ability to reliably and autonomously identify unused frequency bands plays an extremely important role in cognitive radio networks. Relying on the spectrum sensing, ongoing licensed operation must not be compromised and the secondary spectrum usage efficiency should be maintained. Thus, it is critical to ensure that the confidence level of the estimated signal status satisfies the primary user's requirement, whilst keeping the delay and computational complexity to a minimum. This paper provides a comprehensive comparison in terms of performance, reliability and complexity of stand alone sensing schemes for various cognitive radio application areas. We first give some new results on reliability performance, and then evaluate the sensing time required to achieve the target performance. Finally, we compare the computational complexity of various sensing approaches by calculating the number of arithmetic operations required by each approach.
\end{abstract}

\section{INTRODUCTION}

Currently the spectrum resource is managed by national regulators. This fixed and exclusive frequency allotment scheme no longer satisfies the increasing demands of wireless users. In fact, actual spectrum occupancy measurements show that most of the frequency bands below $3 \mathrm{GHz}$ suffer from low average spectrum occupancy even in a dense urban environments[1]. Thus, by allowing opportunistic usage of licensed frequency bands, a huge efficiency improvement can be expected and yield a benefit due to the favourable propagation characteristics of, for instance, TV frequency bands.

Unlicensed users or secondary users, have lower priority of usage of a specific part of the spectrum. Hence, the secondary users need to have a knowledge of the usage of the spectral bands by the licensed (primary) users. Until now, various spectrum sensing algorithms have been proposed in the literature [2]. The FCC in the US has already allocated licences to several white space database systems and the UK is currently testing such a system, both of these do not use sensing. However, sensing systems could be incorporated with the database systems in the future to improve performance.

In this paper, the goal is to analyse and compare the performance of various existing stand-alone spectrum sensing algorithms. In particular, these spectrum sensing approaches are examined in terms of reliability, delay 


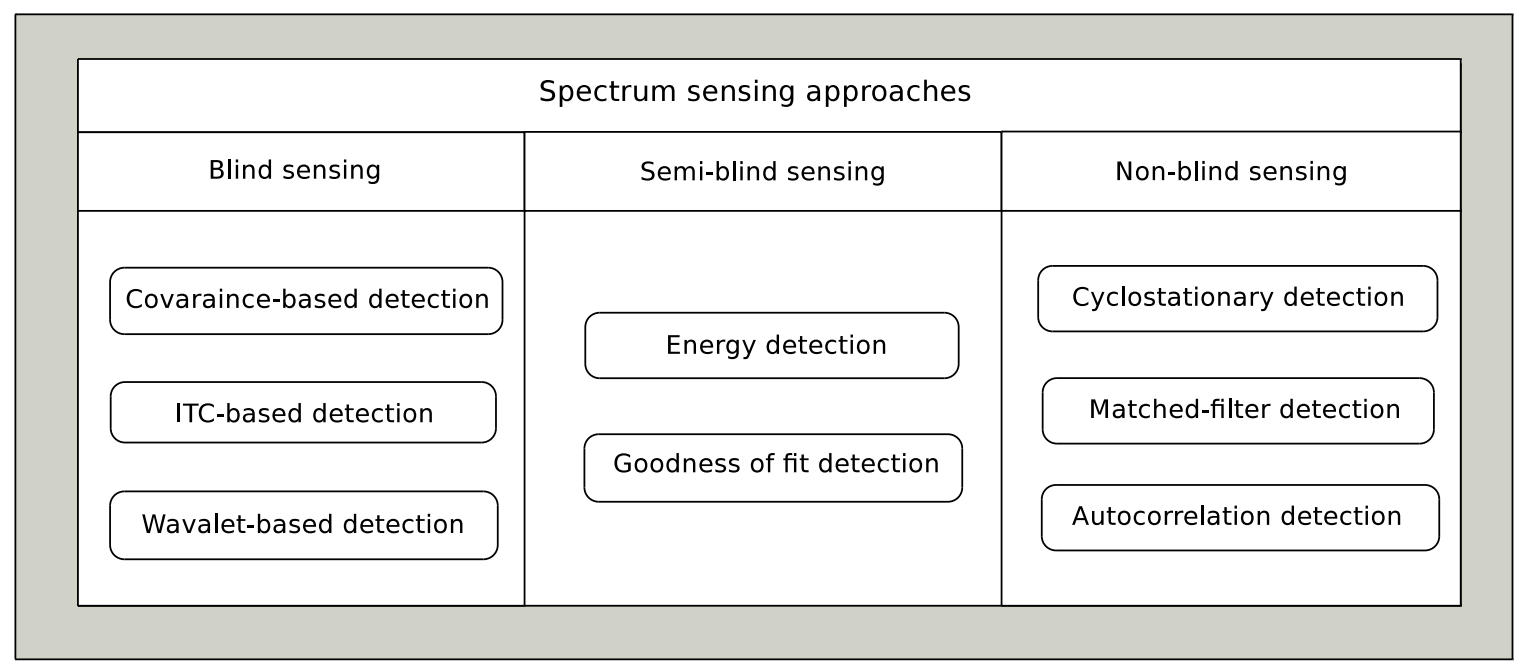

Fig. 1. Classification of the most commonly used stand alone spectrum sensing approaches

and complexity. We first evaluate the impact of different channel environments on the reliability of these detection methods and then investigate the effect of different primary signal structures. To enhance the sensing performance, more signal samples are needed. However, longer sensing times decrease the time available for secondary data transmission. Thus we give a theoretical analysis of the number of signal samples required by each sensing methods in order to achieve a target sensing performance. Also, battery-powered cognitive radios demand low energyconsumption spectrum sensing approaches. The energy consumption of a cognitive radio depends not only on the characteristics of the radio but also on the complexity of the employed sensing method. Therefore, we derive mathematical expressions for the computational complexity of widely used spectrum sensing approaches.

The reminder of this article is organized as follows. The commonly used spectrum sensing techniques are classified and reviewed in Section 2. In Section 3, the most popular spectrum sensing techniques are compared in terms of reliability, delay and computational complexity. Finally, we draw the conclusion in Section 4.

\section{SPECTRUM SENSING TECHNIQUES IN COGNITIVE RADIO NETWORKS}

In this section, we review the most commonly used spectrum sensing techniques used in cognitive radio networks, as shown in Fig. 1. The sensing techniques can be classified into three categories according to the amount of information they require: blind, semi-blind or non-blind.

\subsection{Blind Sensing}

Blind detection refers to detection schemes that require no information about the primary system and the noise distribution. Among existing blind detection schemes, wavelet-base detection is proposed to provide a fast but coarse wideband spectrum detection with the aid of edge detection [3]. Hence, wavelet-based detection is often preferred to be selected as the first stage of a multi-stage detection scheme. In addition, information theoretic criteria (ITC)-based detection calculates the similarity between the distribution of the received signal and that of the additive White Gaussian Noise and is thus able to detect the occupied frequency bands [4]. Furthermore, the 
eigenvalue-based detection exploits the difference between the covariance matrices of the correlated signal and the independent noise to deliver highly reliable spectrum sensing [5]. A critical point is that blind detection schemes cannot differentiate the primary signal form the interference.

\subsection{Semi-blind Sensing}

Semi-blind detection schemes require noise information from learning or training. The most well-known and widely used spectrum sensing approach developed under this category is energy detection [6]. Energy detection simply measures the energy summation of the received signal in either the time or frequency domain. However, its performance is limited by the SNR wall due to noise or/and system uncertainty. Goodness of fit test (GFT) detection incurs similar performance degradation. The GFT detection calculates the discrepancy between the distribution of the observed samples and the distribution of the samples expected under noise conditions which is empirically estimated [7], [8]. Hence, the GFT detection is robust to non-Gaussian noise.

\subsection{Non-blind Sensing}

Given the knowledge of the primary signal, non-blind sensing can be employed and is able to offer more robust sensing performance, provided there is good timing and frequency synchronization. Matched-filter coherent detection is optimal in the sense of completely known data sequence detection [9]. However, it is very sensitive to frequency offset. Instead, second order cyclostationarity can be employed to detect the periodicity of the primary signal statistics at the cost of increased complexity, long latency, and high sensitivity to sampling error [10]. Furthermore, the autocorrelation detection scheme exploits the non-zero average autocorrelation at a time displacement in the signal to provide flexible and reliable spectrum sensing [11], [12].

\section{Comparison of Stand Alone Spectrum Sensing Techniques}

Table 1 provides a brief summary of the most popular standalone spectrum sensing techniques in terms of their most applicable application scenarios. Of these techniques, some make use of primary signal information and thus they fail to operate properly if the primary information is not available; some are susceptible to imperfect channel conditions and estimation error; and some explore a weak feature of the primary signal and then an unreasonable long sensing time is required, etc. In order to capture and address these problems, we compare the performance of these techniques using three metrics: reliability and accuracy, delay and computational complexity.

\subsection{Reliability and Accuracy}

\subsubsection{Effect of Channel Conditions}

In this section, the goal is to investigate the effects of different channel conditions on the performance of various stand alone spectrum sensing schemes. A DVB-T OFDM signal is employed as the primary signal whose parameters are as follows: the number of subcarriers $N_{\underline{F T}}=8192$ of which $N_{\underline{o c c}}=6817$ are occupied, and the cyclic prefix (CP) length $N_{c p}=1024$. The subcarrier modulation is 64QAM, the carrier frequency is $750 \mathrm{MHz}$, and the bandwidth 
TABLE 1

Summary of most popular spectrum sensing techniques in terms of applicable application scenarios

\begin{tabular}{|c|c|c|}
\hline Spectrum sensing scheme & Applicable application scenarios & Requirement \\
\hline Energy detection & $\begin{array}{l}\text { simple, low complexity, } \\
\text { no information about the primary signal, } \\
\text { medium or high SNR }\end{array}$ & known noise power \\
\hline Cyclostationary detection & $\begin{array}{l}\text { signal period is known, } \\
\text { primary system is not sensitive to large time delay }\end{array}$ & powerful processor \\
\hline Matched-filter detection & $\begin{array}{l}\text { simple, low complexity, } \\
\text { complete signal sequence is known, } \\
\text { a short sensing time is required even under very low SNR }\end{array}$ & $\begin{array}{l}\text { synchronization, } \\
\text { dedicated receiver for each primary signal }\end{array}$ \\
\hline Covariance-based detection & $\begin{array}{l}\text { no information is available (neither primary signal nor noise), } \\
\text { multi-path fading }\end{array}$ & $\begin{array}{l}\text { oversampling } \\
\text { or multiple receivers }\end{array}$ \\
\hline Wavelet-based detection & $\begin{array}{l}\text { no frequency boundary information is known, } \\
\text { fast but coarse detection is acceptable }\end{array}$ & $\begin{array}{l}\text { suitable wavelet function } \\
\text { known noise power spectral density }\end{array}$ \\
\hline
\end{tabular}

is $6 \mathrm{MHz}$. For multipath Rayleigh fading, ETSI EN 300744 V1.6.1 (2009-01) Rayleigh fading was used [13]. In additional, the Doppler spread due to the relative motion between the transmitter and the receiver is introduced to both frequency flat and frequency selective Rayleigh fading channels. Shadowing fading is characterized by the shadowing dB-spread, $\sigma_{\underline{d B}}$. The simplest time-domain energy detector is used in the simulation [14]. The method used to measure the goodness of fit is the Kolmogorove-Smirnov (K-S) test [8]. The matched-filter detector utilizes the known pilot pattern of the primary signal to detect the target signal. Due to the $\mathrm{CP}$ nature of the OFDM techniques, both the autocorrelation and cyclostationary detector exploit the $\mathrm{CP}$ to perform the detection. Meanwhile, the test statistic of the cyclostationary detection is derived by the generalized likelihood ratio test (GLRT) [10]. In the following, all the cyclostationary detectors are GLRT cyclostationary detectors. The covariance-based detector employs two independent antennas and the ratio of the maximum eigenvalue to the minimum eigenvalue (MME) is adopted as the test statistic [5].

Fig. 2 indicates that the multipath channel has little impact on the energy and the GFT detector due to a wide channel bandwidth (larger than the coherence bandwidth). Meanwhile, both detectors are insensitive to the time selectivity of the channel since the channel coherence time is larger than the detection time even at very high mobile speeds. For coherent matched-filter detection, the frequency selective fading channel introduces independent channel taps and thus completely eliminates the coherent signal processing gain. For the same reason as with the energy detection and the GFT detection, there is negligible performance loss caused by the Doppler spread. To detect the OFDM signals, both the autocorrelation detection and the cyclostationary detection exploit the CP. Thus, the two detectors exhibit similar performance. Since the employed cyclic frequency $1 / T_{\underline{\underline{s}}}$ is smaller than the channel coherence bandwidth, where $T_{\underline{s}}=N_{\mathrm{FFT}}+N_{\underline{c} \underline{ }}$ is the symbol length, the detectors are relatively insensitive to the 


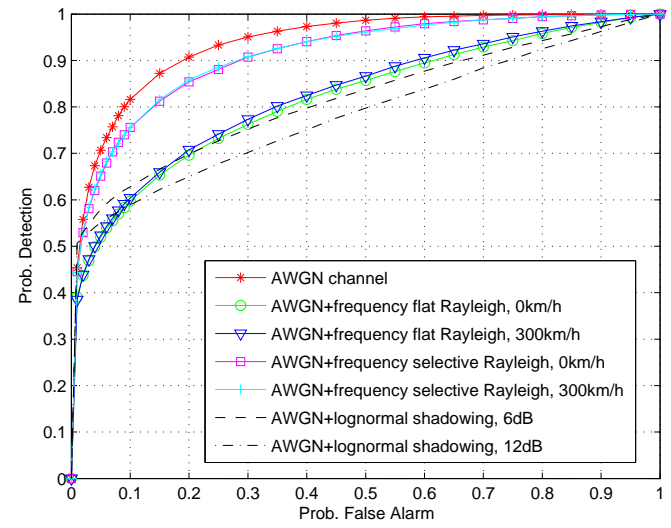

(a) energy detection

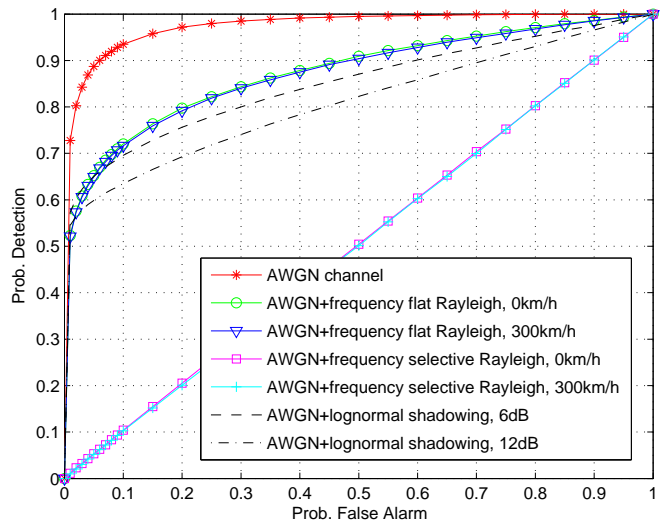

(c) matched-filter detection

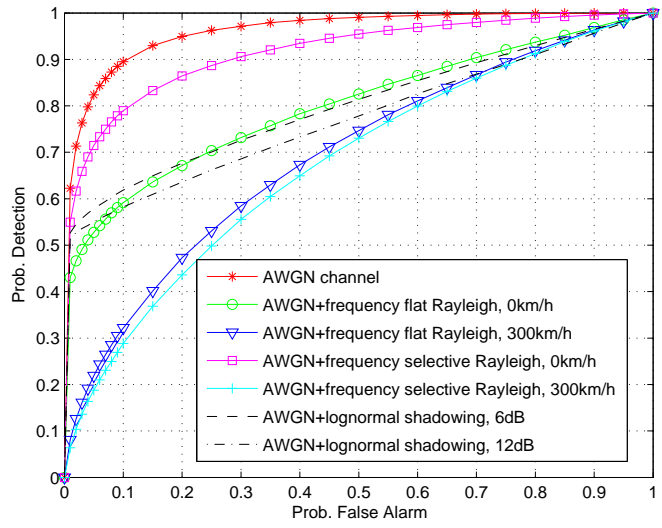

(e) GLRT cyclostationary detection

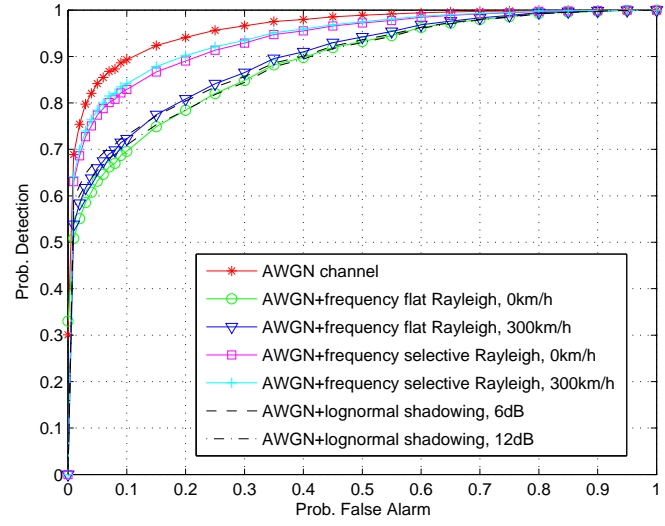

(b) GFT detection

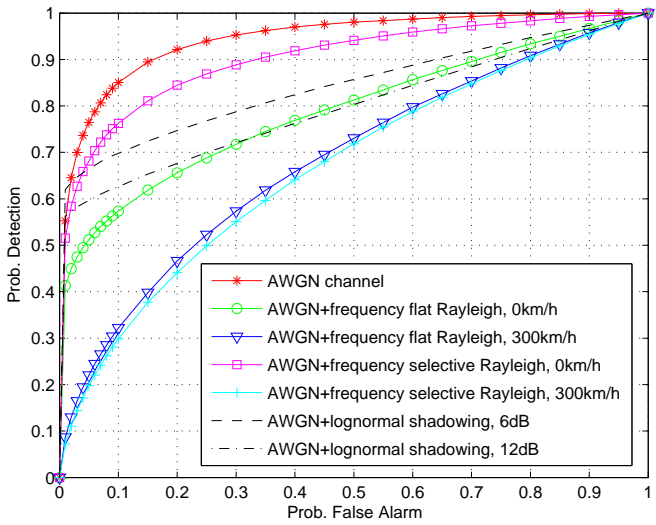

(d) autocorrelation detection

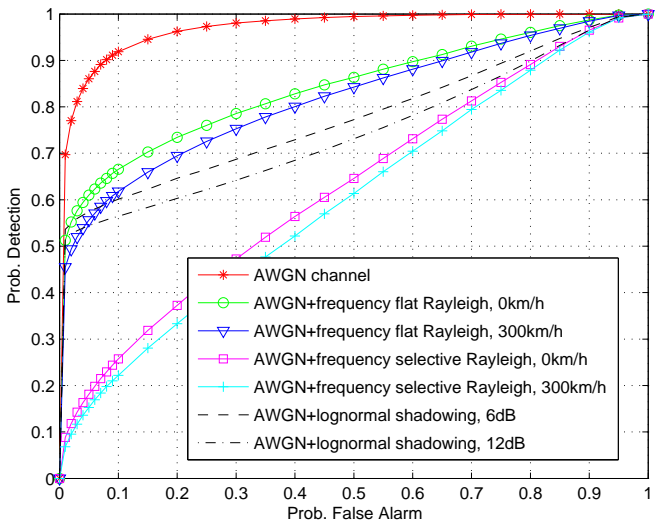

(f) covariance-based detection

Fig. 2. Performance of various spectrum sensing schemes under different channel conditions, $S N R=-15 d B$

frequency shift effects. However, they suffer greatly from the Doppler spread due to long time lag $T_{\underline{d}}=N_{\mathrm{FFT}}$. Thus, for autocorrelation and cyclostationary detection, one should choose small cyclic frequencies and time lags. The spatial correlation of primary signal samples across the antennas can be destroyed by the fading channels. Thus, the covariance-based detector exhibits much worse performance in the Rayleigh fading channel than in AWGN. 


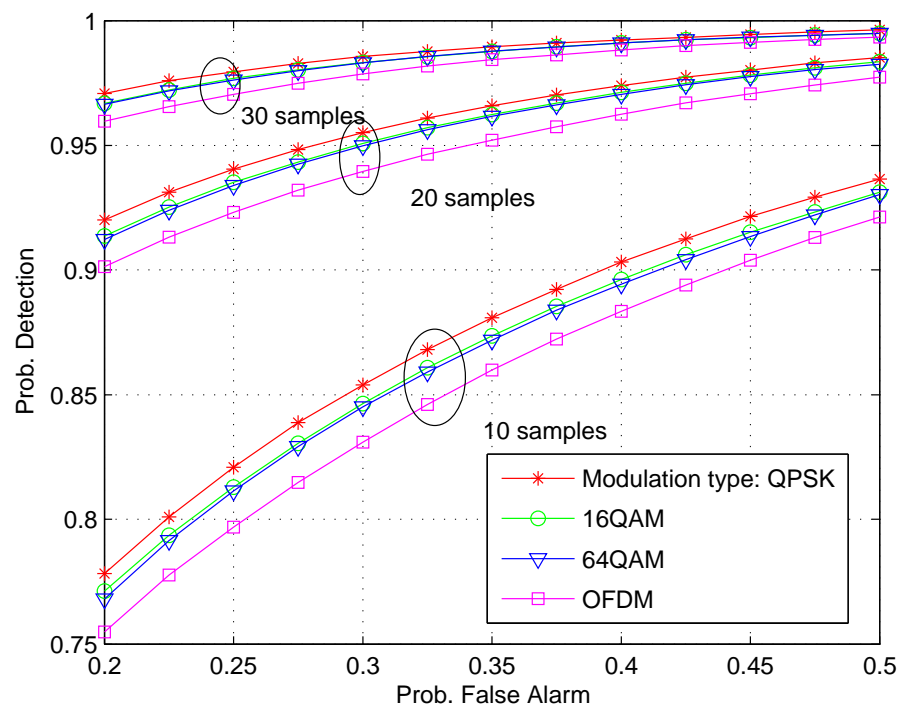

Fig. 3. Performance comparison of energy detection for different modulation type with increased number of samples.

The channel gains of the static frequency-flat Rayleigh channel and the large-scale shadowing fading channel are specific realizations of the corresponding random variables. Thus, the channel gain is time invariant during the sensing period. Observations obtained from the simulations indicate that such fading channels have similar effects on the performance of different sensing techniques.

\subsubsection{Effect of Non-Environmental Factors}

In this section, we provide simulation results to illustrate how the performance of the various spectrum sensing schemes are affected by non-environmental factors, e.g. primary signal modulation type and symbol length.

In Fig. 3, we compare the detection performance of the energy detector as a function of the probability of false alarm for QPSK, 16QAM, 64QAM, and OFDM signals. The energy detector exhibits best performance when the primary signal is QPSK and degrades for 16QAM, 64QAM, and OFDM. The reason is that the samples of the PSK signal are all transmitted with the same energy. However, the amplitude of more sophisticatedly modulated signals can vary. the average received energy fluctuates and hence results in some performance deterioration. Such performance deterioration can be alleviated by increasing the number of samples as shown in Fig. 3 where the performance gap between different modulated signals tends to vanish as the sensing time increases.

The success of the GLRT-based cyclostationary detection algorithm depends on the accuracy of estimation of the cyclic autocorrelation and the cyclic spectrum. In order to make nonparametric, and consistent cyclic spectrum estimates, a smoothed cyclic peirodogram based estimation method is proposed in[15] where the Kaiser window is used. The Kaiser window has an adjustable parameter $\beta$ which controls how quickly it approaches zero at the edges. Fig. 4 compares the distributions of the statistic test of the cyclostationary detection for OFDM signals using different window functions and different window lengths $L_{w}$ with the theoretical chi-squared distribution curve in (a)noise-only scenario and (b)noise plus signal scenario. In the noise-only scenario, with $L_{\underline{w}} \equiv 513$ the accuracy of the asymptotic distribution is very good. Since there is negligible difference between the curves for different 


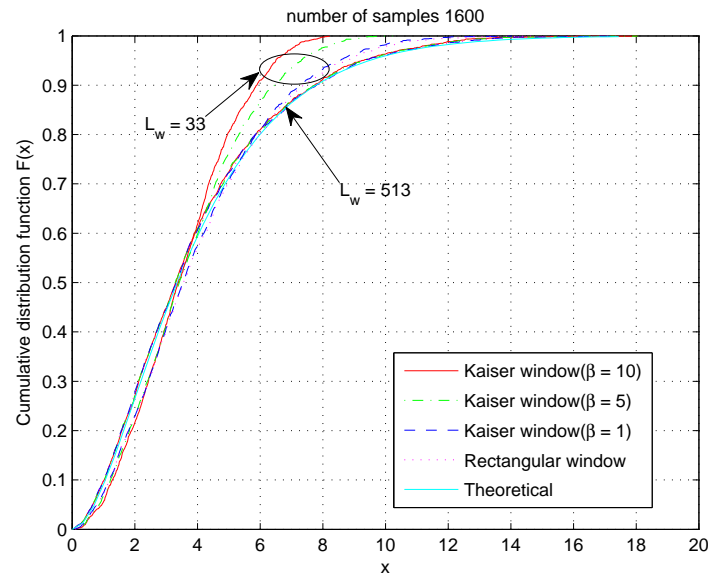

(a) noise only

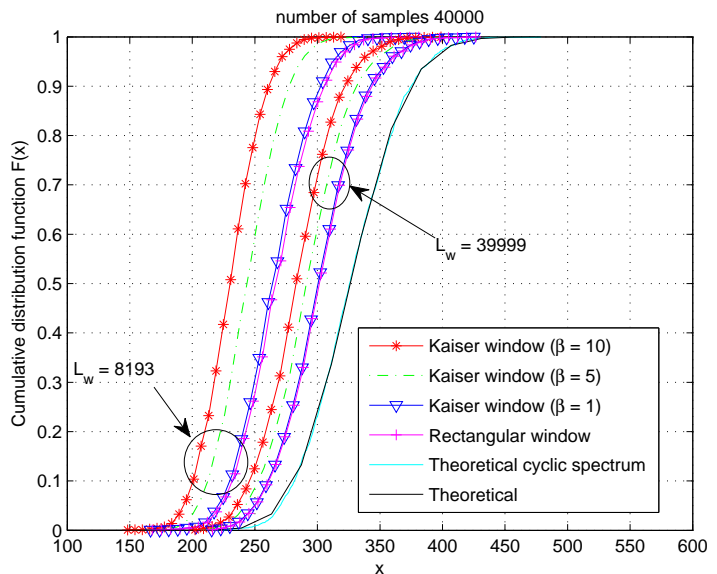

(b) noise+signal, SNR $=-5 \mathrm{~dB}$

Fig. 4. Comparison of the distributions of the statistic test using different window functions with the chi-squared theoretical distribution curve.

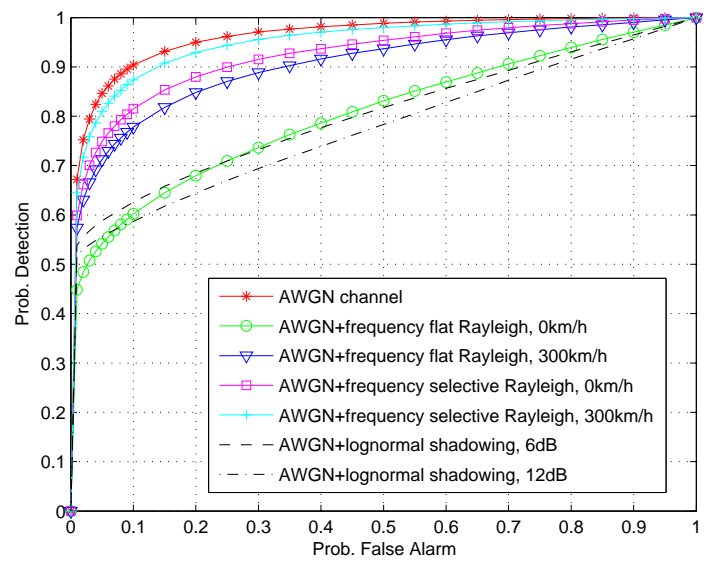

(a) autocorrelation detection

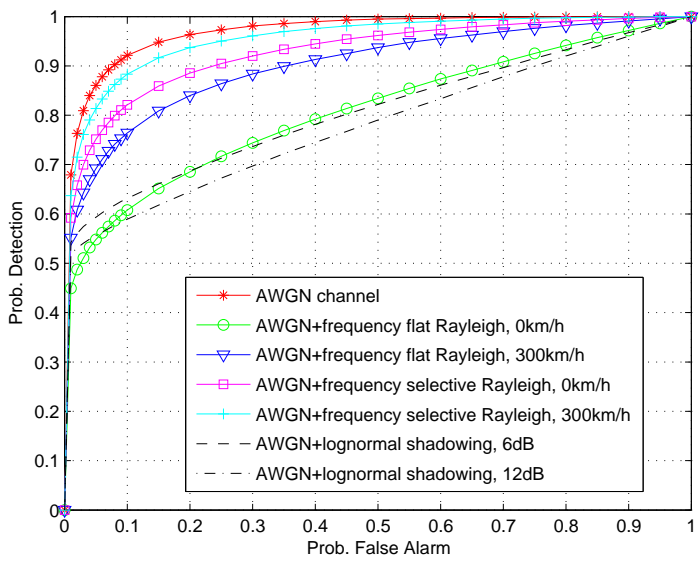

(b) cyclostationary detection

Fig. 5. Performance of CP-based spectrum sensing schemes under different channel conditions, $S N R=-15 d B$

smoothing windows, the smoothing can be efficiently accomplished by using a rectangular window. The figure also shows that the rectangular window performs best when the window length decreases. Fig. 4(b) compares the theoretical curve with the theoretical cyclic spectrum curve which is obtained by using theoretical cyclic spectrum and empirical cyclic autocorrelation in the simulations. The figure shows that the theoretical cyclic spectrum curve hold very accurately with the pure theoretical curve. That is, the number of samples is sufficiently large to accurately estimate the cyclic autocorrelations. However, the figure also shows that the estimation of the cyclic spectrum based on smoothed cyclic peirodogram is not accurate even when very long spectral window is used. Furthermore, the figure confirms the advantage of the simple rectangular window.

Next, we consider the $\mathrm{CP}$-based autocorrelation and cyclostationary detection in fading channels with an OFDM primary signal of shorter symbol length. The primary user signal parameters are as follows: $N_{\text {FFT }} \equiv 512$, and 


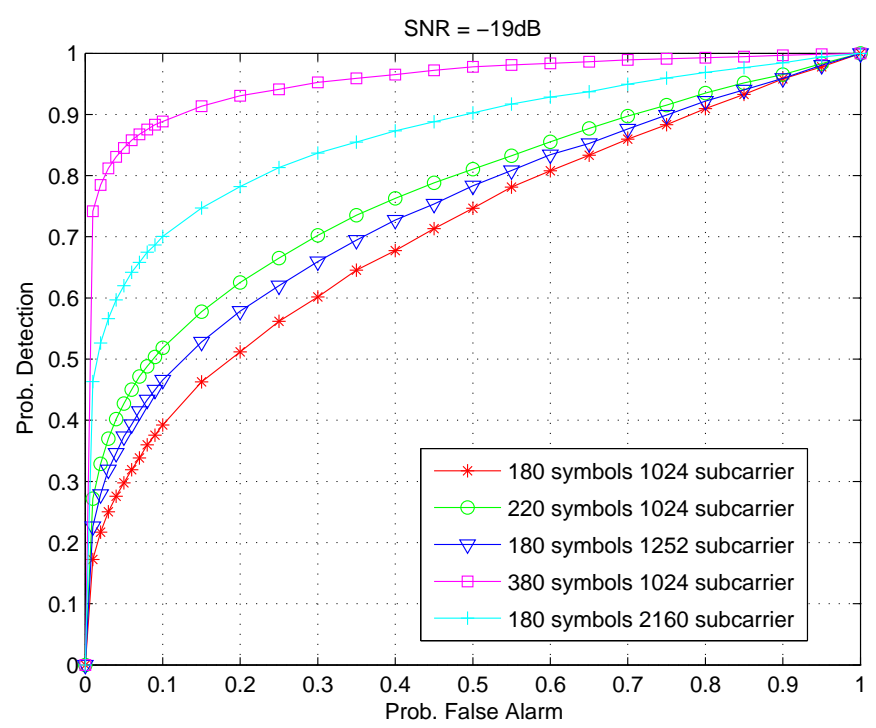

Fig. 6. Performance comparison of TDSC-MRC algorithm using different number of samples, $S N R=-19 d B$

$\underline{N}_{c p}=64$. Fig. 5 shows that the impact of sensor mobility on primary signals with short symbol length is much smaller than that on signals with long symbol length. The reason is that two data sequences are believed to encounter the same channel effect if the time difference between them is short. Thus, the coherent feature of the signal is retained.

Intuitively, a longer sensing time results in a better performance. Fig. 6 shows the performance of a pilot-based autocorrelation detection for the DVB-T OFDM signal[12]. It is shown that for the same number of samples, the detector utilizes a smaller number of sub-carriers and hence a larger number of symbols exhibits better performance. The reason for this is that the performance gain due to incremental number of sub-carriers is less than the performance gain due to the incremental numbers of symbols.

\subsection{Delay}

Different types of detector may need different sensing durations to achieve a given target performance. For the desired $P_{d}$ and $P_{f}$, the minimum number of samples $N$ is a function of the signal to noise ratio SNR. For the time-domain energy detector [14] and when both signal and noise are real-valued Gaussian, $N$ is given by:

$$
N=2\left[\left(Q^{-1}\left(P_{f}\right)-Q^{-1}\left(P_{d}\right)\right)(S N R+1)\right]^{2} S N R^{-2}
$$

where $Q^{-1}(\cdot)$ is the inverse of the standard Gaussian tail probability function. In the low $S N R<<1$ regime, we approximate $S N R+1 \approx 1$ and then the sensing time required scales as, $O\left(S N R^{-2}\right)$.

In practice, it is impossible to have the exact value of the current noise power. Thus assume that the noise variance can take any value within the interval $\left[(1 / \rho) \sigma_{n}^{2}, \rho \sigma_{n}^{2}\right]$, where $\sigma_{n}^{2}$ is the nominal noise power and $\rho$ is a parameter that quantifies the size of the uncertainty. After some manipulation, the minimum number of required samples is approximated by $N \approx\left[Q^{-1}\left(P_{f}\right)-Q^{-1}\left(P_{d}\right)\right]^{2}[S N R-(\rho-1 / \rho)]^{-2}$. It is can be shown that below a certain SNR, the desired $P_{d}$ and $P_{f}$ cannot be met. 
Due to the coherent gain, matched-filter detection [9] requires the minimum possible number of samples. If the pilot signal is BPSK modulated and the data signal and the noise are real-valued Gaussian, then:

$$
N=\left[Q^{-1}\left(P_{f}\right)-\sqrt{1+(1-\theta) S N R} Q^{-1}\left(P_{d}\right)\right]^{2}(\theta \cdot S N R)^{-1}
$$

where $\theta$ is the fraction of total signal power allocated to the pilot tone. When there is no fading, the matched filter is robust to noise uncertainty. The performance limitation is caused by the lack of perfect synchronization. In order to remedy the frequency offset effects, we have to process the received signal, block by block the length of which is equal to the coherence time $N_{c}$. Hence, $N$ is approximated by:

$$
N \approx 2 N_{c}\left[Q^{-1}\left(P_{f}\right)-Q^{-1}(P d)\right]^{2}\left[N_{c} \cdot \theta \cdot S N R-(\rho-1 / \rho)\right]^{-2}
$$

From the above equation, it can be seen that the coherent processing increases the received SNR by $N_{c}$ but a SNR wall is introduced by noncoherent averaging.

Since the symbol timing information is not present, the matched-filter detector has to perform the original matched-filter function at all possible time instances . Furthermore, it is known that if there is a sampling clock offset, there will be a cumulative drift in the position of the sampling points. Such impairments can be mitigated by slicing the total sensing time into several time slots within which the sampling offset is negligible. Assuming that the length of the time slot is much longer than the coherent block length $N_{c}$ and $S N R<<1$, the minimum number of samples of the timing-recovered block-based matched-filter detector is approximated by:

$$
N \approx \frac{2 N_{c}\left[Q^{-1}\left(1-\left(1-P_{f}\right)^{1 / M}\right)-Q^{-1}(P d)\right]^{2}}{\left[N_{c} \cdot \theta \cdot S N R-(\rho-1 / \rho)\right]^{2}}
$$

where $M$ is the maximum time offset. Therefore, timing mismatch introduces additional sensing delay.

For autocorrelation detection [11] with perfect noise and timing knowledge, its sample complexity is given by:

$$
N=\frac{N_{r}+N_{d}}{N_{c}} \frac{\left[Q^{-1}\left(P_{f}\right)-\sqrt{(S N R+1)^{2}+S N R^{2}} Q^{-1}\left(P_{d}\right)\right]^{2}}{S N R^{2}}
$$

where the repeated deterministic data sequence and the noise are both real-valued Gaussian; $N_{r}$ is the length of the repeated sequence and $N_{d}$ is the useful symbol length. When $S N R<<1, N$ scales as $O\left(S N R^{-2}\right)$. One of the advantages of the autocorrelation-based detector is that it is not sensitive to frequency offset, multipath fading channel impairments and noise uncertainty. However, the correct symbol starting point is still unavailable. In order to alleviate the timing offset effect without exhaustive search, the autocorrelation of the entire symbols are calculated as the detection statistic. The resultant sample complexity is given by:

$$
N=\frac{\left[Q^{-1}\left(P_{f}\right)-\sqrt{(S N R+1)^{2}+\left(\frac{N_{c}}{N_{c}+N_{d}} S N R\right)^{2}} Q^{-1}\left(P_{d}\right)\right]^{2}}{\left(\frac{N_{c}}{N_{c}+N_{d}} S N R\right)^{2}}
$$

The test statistic of the GLRT-based cyclostationary detection [10] is Chi-squared distributed under both hypotheses. By approximating the cumulative distribution function (CDF) of the central Chi-squared distributions as the cube of a Gaussian, the sample complexity of the widely used GLRT-based cyclostationary detection in Gaussian noise is given by:

$$
N=\frac{(1+S N R)^{2}\left\{\sqrt{\left[Q^{-1}\left(P_{d}\right)\right]^{2}+2 N_{c a}\left[\frac{\Phi^{-1}\left(1-P_{f}\right)}{3 \sqrt{N_{c a}}}+\left(1-\frac{1}{9 N_{c a}}\right)\right]^{3}}-Q^{-1}\left(P_{d}\right)\right\}^{2}}{2 \cdot \theta^{2} \cdot S N R^{2}}
$$


where $\theta$ is the fraction of total signal power occupied by the employed cyciostationarity, $N_{c a}$ is the number of cyclic autocorrelations, and $\bar{\Phi}^{-\mathrm{I}}(\overline{-})$ is the inverse of the CDF of the standard normai distribution.

After centering and scaling, the distribution of the largest eigenvalue of the received signal sample covariance matrix in the joint limit $K, N \rightarrow \infty$ converges to the Tracy-Widiom distribution of order two and standard Gaussian distribution under $\bar{H}_{0}$ and $\bar{H}_{1}$, respectively, where $K$ is the number of antennas [16]. As for the smallest eigenvalue, its value converges to $\sigma_{\underline{n}}^{2}(\sqrt{K / N}+1)$ aimost surely. Thus, the minimum number of samples required by the maximum to minimum eigenvalue ratio detection [5] for the desired $P_{\underline{d}}$ and $P_{f}$ can be obtained by solving the following equation:

$$
\begin{aligned}
& F_{T W_{2}}^{-1}\left(1-P_{f}\right)\left[\left(\frac{K}{N}\right)^{1 / 2}+1\right]\left[\left(\frac{K}{N}\right)^{-1 / 2}+1\right]^{1 / 3}+\left[\left(\frac{K}{N}\right)^{1 / 2}+1\right]^{2} \\
= & Q^{-1}\left(P_{d}\right)(K S N R+1) \sqrt{N-\frac{1}{K S N R^{2}}}+(K S N R+1)\left(N+\frac{1}{S N R}\right)
\end{aligned}
$$

where $F_{T_{W}}^{-1}(\cdot)$ is the inverse of the CDF of the Tracy-Widom distribution of order two. For such high degree polynomial equations, their roots can in general only be found by numerical methods.

The Kolmogorove-Smirnov (K-S) Test [8] is a non-parametric method to measure the goodness of fit. Though having several limitations, the K-S detector is attractive due to its advantage of having no assumptions on the distribution of noise. For sufficiently large sample size, The sample complexity of the one-sample K-S test detection is approximated by:

$$
N \approx \frac{1}{2}\left[\sqrt{\log \left(\frac{2}{P_{f}}\right)}+\sqrt{\log \left(\frac{2}{P_{d}}\right)}\right]^{2}\left[d_{k}\left(F_{0}, G_{0}\right)\right]^{-2}
$$

where $d_{\underline{k}}(A, B)$ is the maximum vertical distance between distribution $\mathrm{A}$ and $\mathrm{B}$, and $F_{0}$ and $G_{0}$ are the theoretical CDFs of the signal samples under null and alternative hypotheses, respectively. When $F_{0}$ and $G_{0}$ are specified, $\underline{d_{\underline{k}}}\left(F_{\underline{\underline{0}}}, G_{\underline{\underline{0}}}\right)$ is a constant.

Figure 7 illustrates the number of samples required to achieve the desired $P_{f}=0.01$ and $P_{d}=0.99$ for a given $S N R$. The primary user is assumed to be an IEEE 802.11a/g OFDM signal. The number of subcarriers $N_{\mathrm{FFT}}=64$, the number of occupied subcarriers $N_{o c c}=52$, and the cyclic prefix length $N_{C P}=16$. BPSK modulated pilots are inserted every thirteen subcarriers. Figure 7 also illustrates how the required number of samples varies for the energy detector and block-based matched-filter detector when the $S N R$ approaches the SNR wall. The noise uncertainty parameter $\rho$ is set to 1.005 .

It can be seen that the ideal matched-filter detector outperforms the other detectors under low SNR regime. The MME and energy detectors perform worse than the matched-filter detector by some way, but due to the perfectly known or estimated noise variance, the MME and energy detectors outperform the other sensing techniques. Since the autocorrelation detection only utilize $1 / 5$ of the total data sequence for detection, it requires many more data samples than the energy detection. It is clearly shown that the cyclostationary detection requires an order of magnitude more samples than the other detection methods to achieve the target performance.

Both the energy detection and matched-filter detection suffer from the noise uncertainty problems. Their required number of samples becomes infinite when the SNR approaches the SNR wall. Due to the coherent processing the SNR wall of the matched-filter detection is much lower than that of the energy detection. 


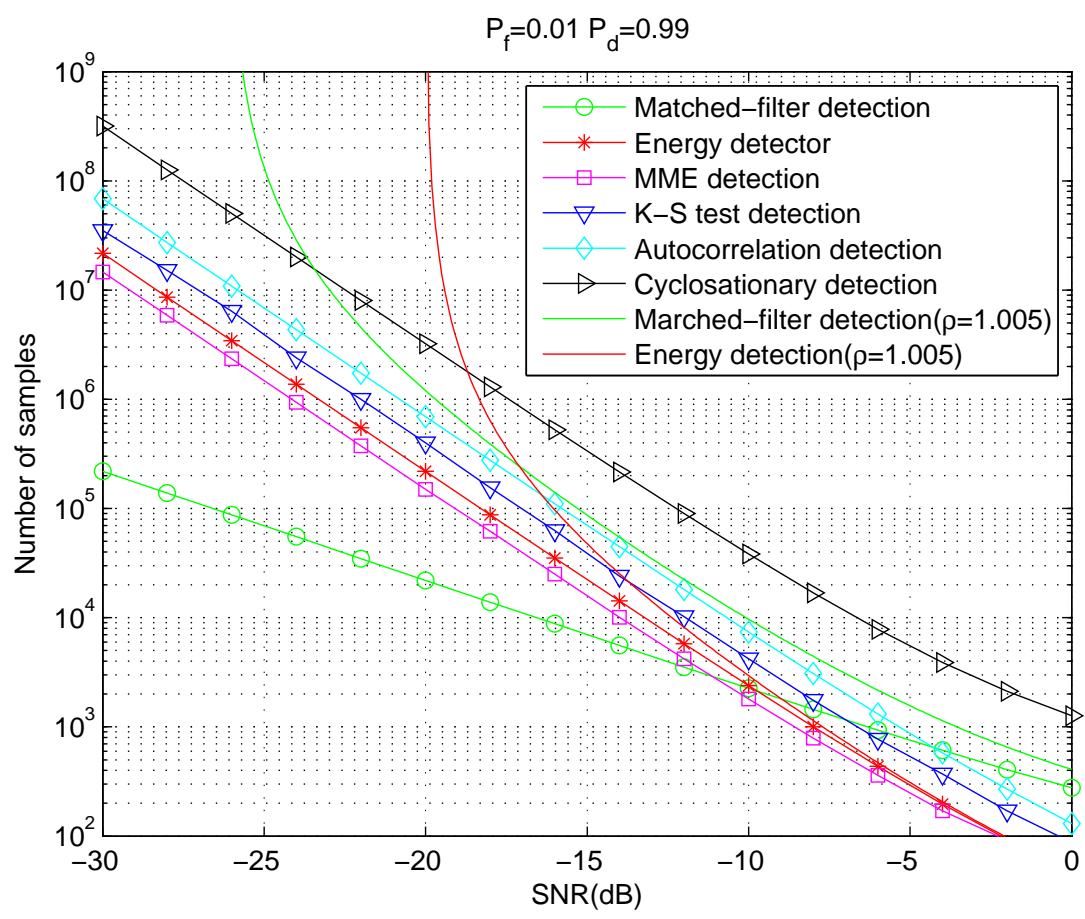

Fig. 7. SNR vs. Number of samples for different detection methods under $\underline{P}_{f}=0.01$ and $\underline{P_{d}}=0.99$ and the worst case for energy detection and block-based matched-filter detection under noise uncertainty.

\subsection{Computational Complexity}

To evaluate the computational complexity for spectrum sensing methods, we count the number of real multiplications (RM), real additions (RA) and comparisons. Other operations such as loading, storing, loop counting, indexing, etc are not counted.

\subsubsection{Energy detection}

Denoting $N_{s}$ the number of received samples, the time-domain energy detection [14] requires $N_{s}$ complex multiplications (CM) and $N_{s}-1$ RAs. If the energy detection is implemented in the frequency domain [17], the computational complexity depends on these parameters: number of FFT point $N_{\mathrm{FFT}}$, number of averaging $N_{a v}$ and the number of sub-channel $N_{c}$. Thus, the energy detection (frequency domain) requires $N_{a v}\left(N_{\mathrm{FFT}}+\frac{N_{\mathrm{FFT}}}{2} \log _{2} N_{\mathrm{FFT}}\right) \mathrm{CMs}$, $N_{a v} N_{\mathrm{FFT}} \log _{2} N_{\mathrm{FFT}}$ complex additions (CA), and $\left(N_{a v}-1\right) N_{\mathrm{FFT}}+\left(N_{c}-1\right) \frac{N_{\mathrm{FFT}}}{N_{c}}$ RAs.

\subsubsection{Matched-filter coherent detection}

The computational complexity of the matched-filter detection [9] is related to the total number of pilot samples $N_{p}$. Hence, pilot-based detection requires $N_{p}$ CMs and $N_{p}-1$ CAs. If frequency offset is considered and the length of the channel coherent time is denoted by $N_{c}$, the detector requires $N_{p}+\frac{N_{p}}{N_{c}} \mathrm{CMs}, N_{p}-\frac{N_{p}}{N_{c}}$ CAs and $\frac{N_{p}}{N_{c}}-1$ RAs. If we further consider the timing offset, the detector requires $M\left(N_{p}+\frac{N_{p}}{N_{c}}\right)$ CMs and $M\left(N_{p}-\frac{N_{p}}{N_{c}}\right)$ CAs, $M\left(\frac{N_{p}}{N_{c}}-1\right)$ RAs and $M-1$ comparisons where $M$ is the maximum time offset. 


\subsubsection{Autocorrelation detection}

Assuming that the total number of the repeated samples is $N_{r}$, the autocorrelation detection [11] requires $N_{r}$ CMs and $N_{r}-1$ CAs. Similarly, autocorrelation detector without perfect timing information requires $N_{s}$ CMs and $N_{s}-1$ CAs where $N_{s}$ is the number of samples.

\subsubsection{Cyclostationary detection}

In the following a quantitative analysis of the complexity of the GLRT-based cyclostationary detection is given. The analysis is based on a classic GLRT-based statistical test derived in [10]. Let's consider a received signal with parameters as number of samples $N_{s}$, the FFT point $N_{\mathrm{FFT}}$, the odd window length $L_{w}$, the number of cyclic frequencies of interest $N_{\alpha}, N_{\tau}=\sum_{n=1}^{N_{\alpha}} N_{\tau_{n}}$ where $N_{\tau_{1}}, \cdots, N_{\tau_{N_{\alpha}}}$ are the number of time lags for each different cyclic frequency.

First, the detector needs to calculate the cyclic autocorrelation function which requires $N_{\tau} N_{s}+N_{\tau} \frac{N_{\mathrm{FFT}}}{2} \log _{2} N_{\mathrm{FFT}}$ CMs, $N_{\tau}$ Real-Complex multiplications (multiply a complex number by a real number: RCM) and $N_{\tau} N_{\mathrm{FFT}} \log _{2} N_{\mathrm{FFT}}$ CAs. Second, we consider the calculation of the asymptotic covariance matrix. The total complexity of the asymptotic covariance matrix is given by $4 L_{w} N_{\tau}^{2} \mathrm{CMs}, 4 N_{r}^{2} \mathrm{RCMs}$ and $2 L_{w} N_{\tau}^{2} \mathrm{CAs}$. In practice, symmetry can be used to reduce the calculation of the covariance matrix.

The final computational complexity is obtained by summing the above two complexities and the extra computational cost due to the matrix multiplication: $4 N_{\tau}^{2}+2 N_{\tau}+1$ RMs and $4 N_{\tau}^{2}-1$ RAs. Note that an additional $O\left(N_{\tau}^{3}\right)$ complexity should also be added due to evaluation of the inverse of the covariance matrix.

\subsubsection{Covariance-based detection}

The computational complexity of the most popular covariance-based detection, MME detection [5], arises mainly from two operations: calculation of the sample covariance matrix and eigenvalue decomposition. Suppose that there are $N_{a n}$ antennas and each antenna receives $N_{s}$ signal samples, the detector requires $N_{a n}\left(N_{a n}+1\right) N_{s} / 2 \mathrm{CMs}$ and $N_{a n}\left(N_{a n}+1\right)\left(N_{s}-1\right) / 2$ CAs to compute the sample covariance. The size of the resultant covariance matrix is $N_{a n} \times N_{a n}$, then at most $O\left(N_{a n}^{3}\right)$ CMs and CAs are needed to decompose the matrix. In practice, $N_{s}$ is usually much larger than $N_{a n}$ and thus the computational complexity of the covariance matrix calculation dominates.

\subsubsection{Kolmogorove-Smirnov test detection}

The K-S test first forms the empirical CDF from the observed signal samples and then derives the largest absolute difference between the empirical and theoretical CDFs. Thus, the detector requires $O\left[\left(N_{\underline{s}}+N_{\underline{b}}\right) \log _{\underline{2}}\left(N_{\underline{s}}+N_{\underline{b}}\right)\right]$. comparisons to form the empirical CDF of length $N_{\underline{b}}$ where $N_{\underline{\underline{s}}}$ is the number of received samples. To calculate the

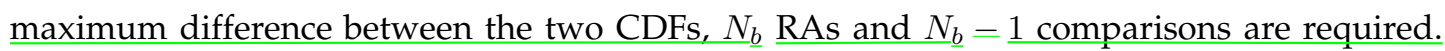

\subsubsection{Wavelet-based detection}

The operational complexity of the wavelet-based detection [3] is caused mostly by the convolution of the wavelet and the PSD of the received signal. Let $N_{w}$ demote the length of the wavelet, then the above operation requires $N_{\mathrm{FFT}} N_{w}$ RMs and $N_{\mathrm{FFT}} N_{w}-N_{\mathrm{FFT}}-N_{w}+1$ RAs. 
TABLE 2

Computational complexity summary of common spectrum sensing techniques

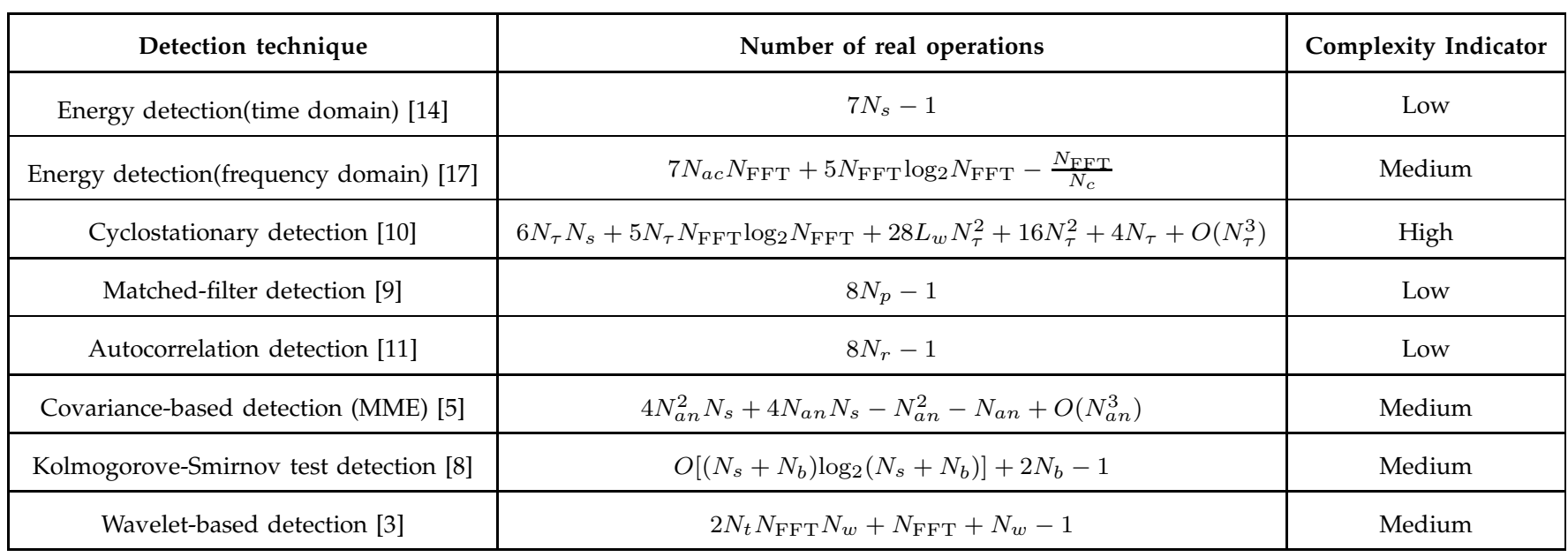

To enhance the multiscale performance while suppressing the noise, the product of wavelet transforms of various dilated versions of the wavelet is used as the final test statistic. Let $N_{t}$ denote the number of accumulated transforms and thus the number of required RMs is $N_{t} N_{\mathrm{FFT}} N_{w}+\left(N_{t}-1\right)\left(N_{\mathrm{FFT}}+N_{w}-1\right)$ and the number of required RAs is $N_{t}\left(N_{\mathrm{FFT}} N_{w}-N_{\mathrm{FFT}}-N_{w}+1\right)$.

With modern DSP, the times consumed by real multiplication, real addition and comparison are the same. Given CM requires four RMs and two RAs, RCM requires two RMs, CA requires two RAs, the numbers of real operations required by different sensing approaches for detecting the primary signal under the assumption of ideal noise and channel condition estimation are given in Table 2.

Denoting $t$ as the unit time required by the cognitive radio to calculate a single RM, a single RA or a signal comparison, we compare the number of unit time required by various popular sensing techniques as a function of SNR in Fig. 8. The simulation parameters are the same as those used in Section 4.2. It is shown that match-filter detection still has the best performance since the detector utilizes the complete information of the primary signal. The autocorrelation detector, although requiring longer sensing time than the energy detector, has better performance than the energy detection with respect to computational complexity. However, the performance difference between cyclostationary detection and other sensing techniques becomes even larger which indicates that large computational complexity is required for cyclostationry detection.

\section{Conclusion}

In this paper, several issues affecting spectrum sensing schemes for cognitive radio networks have been investigated and discussed. We compare some well-known and commonly used sensing methods in terms of reliability and accuracy. We also introduce two important new metrics, the delay and the computational complexity, to compare the performance of these techniques in both analytic and simulation ways. Our comparison is thus more comprehensive than those existing in the current literature which consider fewer types of sensing approaches or lack detailed mathematical analysis or simulation results. 


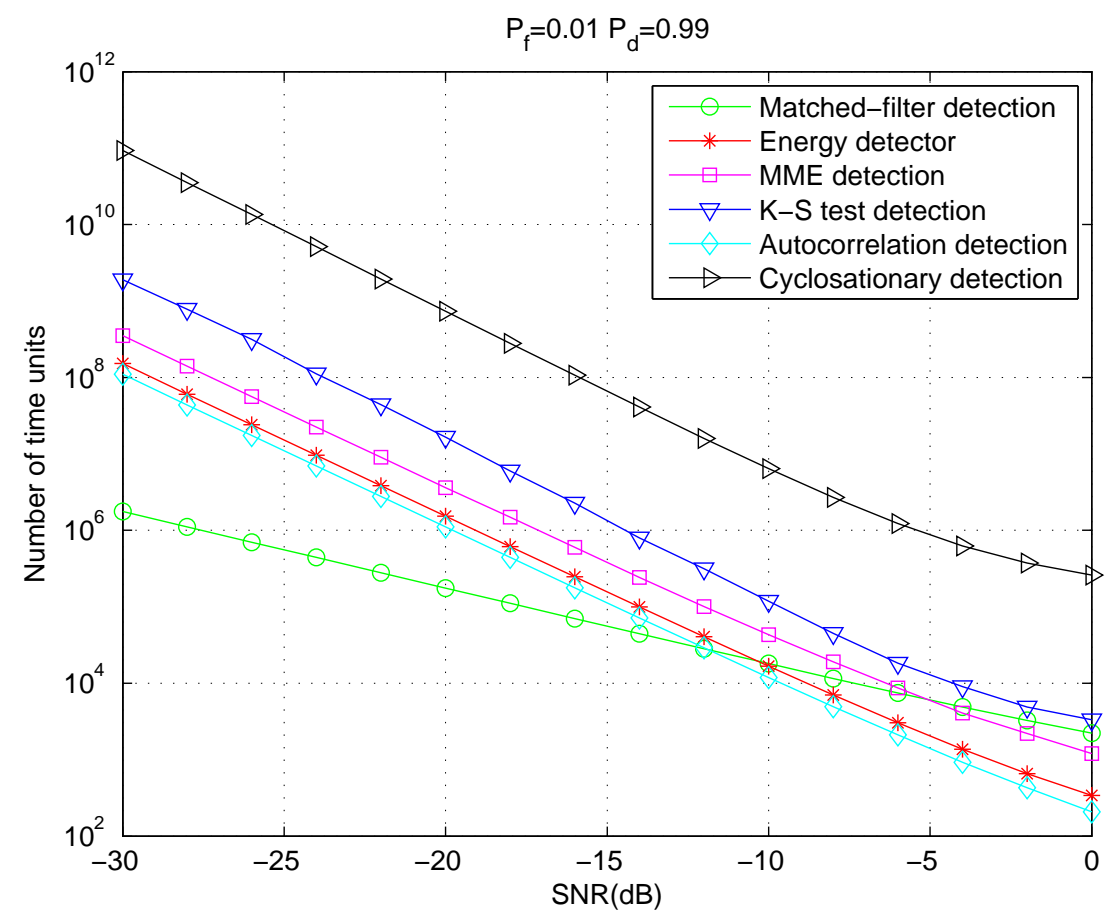

Fig. 8. SNR vs. Computational complexity for different detection methods under $\underline{P}_{f}=0.01$ and $\underline{P}_{d}=0.99$

According to the secondary and primary system requirements, available primary signal information, battery capacity etc., designers of cognitive radio networks should be aware of the most appropriate technique for any specific scenario. Also, based on the work presented in this paper, we can extend research to heterogeneous cognitive radio networks including a mix of sensing nodes. There has been little attention devoted to the problem of selecting and combining sensing results in a heterogeneous network. The performance of different sensing methods obtained in this paper can be used as parameters to design such heterogeneous systems and this is the subject of ongoing research.

\section{ACKNOWLEDGMENT}

The authors would like to thank the EPSRC/DST IU-ATC next generation networks program which partly funded this research.

\section{REFERENCES}

[1] A. Shukla, "Cognitive radio technology - a study for Ofcom," Tech. Rep. 830000143, QinetiQ Ltd, Hampshire, UK, 2006

[2] T. Yucek and H. Arslan, "A survey of spectrum sensing algorithms for cognitive radio applications," IEEE Commun. Surveys and Tutorials, vol. 11, no. 1, pp. 116-130, First Quarter. 2009.

[3] Z. Tian and G. B. Giannakis, "A wavelet approach to wideband spectrum sensing for cognitive radios," in Proc. IEEE CROWMCOM, Mykonos Island, Greece, Jun. 2006., pp.1-5.

[4] B. Zayen, A. M. Hayar and D. Nussbaum, "Blind spectrum sensing for cognitive radio based on model selection," in Proc. IEEE CROWNCOM, Singapore, May 2008, pp.1-4.

[5] Y. Zeng, Y. Liang, "Eigenvalue-based spectrum sensing algorithms for cognitive radio," IEEE Trans. Commun., vol.57, no.6, pp.1784-1793, Jun. 2009. 
[6] H. Urkowitz, "Energy detection of unknown deterministic signals," Proceedings of the IEEE, vol. 55, pp. 523-531, 1967.

[7] H. Wang, E. -H. Wang, Z. Zhao and W. Zhang, "Spectrum sensing in cognitive radio using goodness of fit testing", IEEE Trans. Wireless Commun.,vol. 8, no. 11, pp. 5427-5430, Nov. 2009.

[8] G. Zhang, X. Wang, Y. -C. Liang and J. Liu, "Fast and robust spectrum sensing via Kolmogorov-Smirnov test," IEEE trans. Commun., vol. 58, no. 12, pp. 3410-3416, 2010.

[9] H. S. Chen, W. Gao, and D. G. Daut, "Signature based spectrum sensing algorithms for IEEE 802.22 WRAN," in Proc. IEEE ICC,Glasgow, U.K., Jun. 2007, pp. 64876492.

[10] A. V. Dandawate and G. B. Giannakis, "Statistical tests for presence of cyclostationarity," IEEE Trans. Signal Process., vol. 42, no. 9, pp. 23552369, Sep. 1994.

[11] S. Chaudhari, V. Koivunen, and H. V. Poor, Autocorrelation-based decentralized sequential detection of OFDM signals in cognitive radios, IEEE Trans. on Signal Process., vol. 57, no. 7, pp. 26902700, Jul. 2009.

[12] H. shin Chen, W. Gao and D. Daut, "Spectrum sensing for OFDM systems employing pilot tones," IEEE Trans. Wireless Commun., vol. 8, no. 12 , pp. 5862-5870, 2009.

[13] European Telecommunications Standards institute, Digital Video Broadcasting (DVB), Framing Structure, Channel Coding and Modulation For Digital Terrestrial Television ETSI EN 300744 V1.6.1 (2009-01). jan. 26, 2009[Online]. Available: http:/webapp.etsi.orgworkprogramReport_WorkItem.asp?WKI_ID=28813

[14] F. F. Digham, M. S. Alouini, and M. K. Simon, "On the energy detection of unknown signals over fading channels," IEEE Trans. Commun., vol. 55, no. 1, pp. 2124, 2007.

[15] B. M. Sadler and A. V. Dandawate, "Nonparametric estimation of the cyclic cross spectrum," IEEE Trans. Inform. Theory, vol. 44, no. 1, pp. 351-358, Jan. 1998.

[16] F. Penna and R. Garello, "Theoretical performance analysis of eigenvalue-based detection." Available: http://arxiv.org/abs/0907.1523

[17] J. Ma, G. Y. Li and B. H. Juang, "Signal Processing in Cognitive Radio," Proceedings of the IEEE, vol. 97, no. 5, pp. 805-823, 2009. 